\title{
Cloning, expression and enzyme activity delineation of two novel CANT1 mutations: the disappearance of dimerization may indicate the change of protein conformation and even function
}

\author{
Hong-Dan Wang ${ }^{1,2,3,4^{*}}$, Liang-Jie Guo ${ }^{1}$, Zhan-Qi Feng ${ }^{5}$, Da-Wei Zhang ${ }^{6}$, Meng-Ting Zhang ${ }^{1}$, Yue Gao ${ }^{1}$,
} Chuan-Liang Chen ${ }^{1}$ and Bo-Feng Zhu ${ }^{3,4^{*}}$

\begin{abstract}
Background: Desbuquois dysplasia (DBQD) was a rare autosomal recessive skeletal dysplasia. Calcium activated nucleotidase 1 (CANT1) mutation was identified as a common pathogenic change for DBQD type 1 and Kim variant but not for DBQD type 2. To our knowledge, all patients with DBQD type 1 currently found could be explained by mutations in the CANT1 gene, but mutations in the CANT1 gene might not be directly diagnosed as DBQD type 1.

Results: We have identified two novel CANT1 mutations (mut1: c.594G > A [p.Trp198*], mut2: c.734C > T [p.Pro245Leu]) in three children from a family of Chinese origin for the first time. Two of the three children could be diagnosed as typical DBQD type 1 and one child could not be diagnosed as DBQD type 1 based on the clinical data we had. To further clarify the effect of the two mutations of the CANT1 gene, we studied the CANT1 gene expression and detected the protein secretion and nucleotide enzyme activity through cDNA cloning and expression vectors construction for wild and mutant types. The mut1 was a nonsense mutation which could lead to premature termination and produced the truncated bodies; The CANT1 dimer of mut2 was significantly reduced and even undetectable. The extracellular secretion of mut1 was extremely high while mut2 was significantly reduced compared with the wild type. And mut1 and mut2 also could result in a significant reduction in the activity of CANT1 nucleotidease. From the results we could deduce that the two mutations of the CANT1 gene were the causes of the two cases in this study.

(Continued on next page)
\end{abstract}

* Correspondence: wanghongdan5495@163.com; zhubofeng7372@126.com

${ }^{1}$ Medical Genetic Institute of Henan Province, Henan Provincial People's

Hospital, Zhengzhou University People's Hospital, Zhengzhou 450003,

People's Republic of China

${ }^{3}$ Clinical Research Center of Shaanxi Province for Dental and Maxillofacial

Diseases, College of Stomatology, Xi'an Jiaotong University, Xi'an 710004,

People's Republic of China

Full list of author information is available at the end of the article

(c) The Author(s). 2020 Open Access This article is licensed under a Creative Commons Attribution 4.0 International License, which permits use, sharing, adaptation, distribution and reproduction in any medium or format, as long as you give appropriate credit to the original author(s) and the source, provide a link to the Creative Commons licence, and indicate if changes were made. The images or other third party material in this article are included in the article's Creative Commons licence, unless indicated otherwise in a credit line to the material. If material is not included in the article's Creative Commons licence and your intended use is not permitted by statutory regulation or exceeds the permitted use, you will need to obtain permission directly from the copyright holder. To view a copy of this licence, visit http://creativecommons.org/licenses/by/4.0/. The Creative Commons Public Domain Dedication waiver (http://creativecommons.org/publicdomain/zero/1.0/) applies to the data made available in this article, unless otherwise stated in a credit line to the data. 
(Continued from previous page)

Conclusions: Regarding the particularity of the cases reported in this study, the pathogenesis of CANT1 might be more complicated. The genetic and phenotype of three children with the same genetic background need to be further studied. Larger cohort of patients was needed to establish genotype-phenotype correlations in DBQD.

Keywords: Desbuquois dysplasia, CANT1, Novel mutation

\section{Background}

Desbuquois dysplasia (DBQD, OMIM\#251450) was a rare autosomal recessive genetic disease with the incidence of approximately $1 / 1000000$ and a lowest lethality rate of about $1 / 3[1,2]$. In 1966, the clinical symptoms were first described by Desbuquois et al. [3]. The clinical symptoms of DBQD could be summarized as prenatal and postnatal growth retardation, short stature, multiple joint dislocations, scoliosis/lordosis, short long bones and hand abnormalities. Characteristic radiological features included advanced carpal bone age and a "Swedish key" appearance of the proximal femur. Additional anomalies of hand included distal phalangeal bifurcation or the delta phalanx of the first finger, accessory ossification center distal to the second metacarpal and phalangeal dislocations $[4,5]$.

In 2004, DBQD was classified into Type 1 (hand abnormalities present) and Type 2 (hand abnormalities absent) according to whether the above typical hand abnormalities occurs [6]. In 2010, Kim variant of DBQD has been distinguished characterized as having advanced carpal bone age, short metacarpals and elongated phalanges. The hand and spine abnormalities were the major clinical symptoms of this Kim variant [7]. The disease gene of DBQD was first located on chromosome $17 q 25.3$ by a genome-wide research in four DBQD families with DBQD type 1 patients in 2003 [8]. Until 2009, calcium-activated nucleotidase 1 (CANT1) mutations were first identified in patients of DBQD type 1 [9]. In the studies after 2009, CANT1 mutations were supported as the causes of DBQD type 1. The studies also found that the CANT1 mutations were responsible for Kim variant and atypical DBQD type $2[4,10]$. For DBQD type 2, xylosyltransferase 1 (XYLT1) mutations were found in some of the patients. There were still some unknown molecular bases of DBQD type 2 [11, 12]. To summarize, CANT1 mutation was a common pathogenic change for DBQD type 1 and Kim variant but not for DBQD type 2 .

At present, we identified two novel CANT1 mutations (compound heterozygous) in three children from a family of Chinese origin. According to their clinical symptoms, two of the three children could be diagnosed as typical DBQD type 1 . The other child only had height restrictions with a height of $142 \mathrm{~cm}$ in her 16 years who couldn't be diagnosed as DBQD based on the clinical data. This study found the genetic heterogeneity of CANT1 which has not been reported in previous studies. Through the in vitro experiments by synthesizing fulllength wild-type and mutant CDNA of CANT1 gene, this research still supported the previous findings that CANT1 mutations were responsible for DBQD type 1 . The results of our in vitro experiments showed that the disappearance of dimerization of the mutant protein might be a change affecting CANT1 enzyme activity.

\section{Results \\ Clinical report}

The parents of the patients came to the clinic of the medical genetics institute of Henan Provincial People's Hospital for genetic consultation in order to have guidance to aristogenesis and good brood. The parents were pregnant three times and gave birth three times in full term. The clinical phenotype of parents was normal. The clinical manifestations and radiological findings of the 3 children were showed in Table 1. The first child (child 1) was only limited in height (142 cm, Female, 16 years) and had normal intelligence. And she had no clinical symptoms such as joint dislocation and heart function limitation since birth. The second child (child 2) had pre and postnatal growth retardation, dislocation of joint, lumbar vertebra scoliosis, Mitral valve prolapse with severe insufficiency, camptodactyly, claw toes and hammer toes (Fig. 1a and b). At the age of 3 years, the operation was performed because of congenital dislocation of both hip joints and postoperative recovery was well. At the age of 7 years and 10 months, "mitral valvuloplasty" was performed for mitral valve prolapse with insufficiency found in physical examination and postoperative recovery was well. The third child (child 3) had pre and postnatal growth retardation, dislocation of joint, mitral insufficiency, atrial septal defect, camptodactyly, claw toes and hammer toes (Fig. 1c and d). From her 16 months to 2 years old, the operations were performed due to dislocation of hip joint and postoperative recovery was well. At the age of 2 years and 11 months, mitral valvuloplasty and atrioseptopexy was performed for mitral insufficiency and atrial septal defect. We retrieved radiographs of the hip dislocation of the third child before surgery. Hip dislocation and typical Swedish key appearance could be observed (Fig. 2). We reviewed the Xray of her hands and feet at her 8 years old (Fig. 3). The 
Wang et al. Orphanet Journal of Rare Diseases

(2020) 15:240

Page 3 of 13

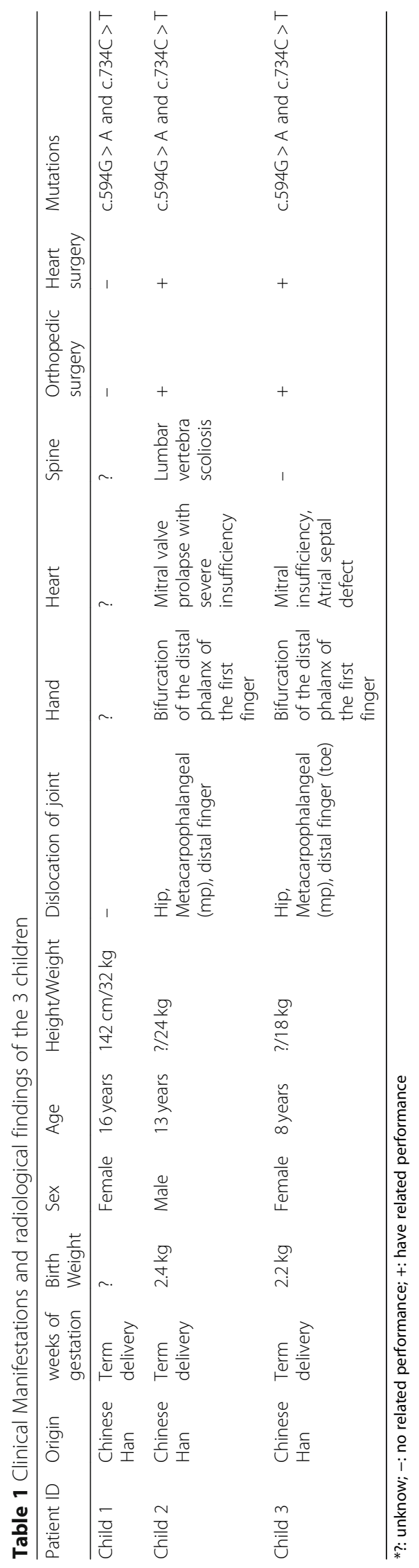



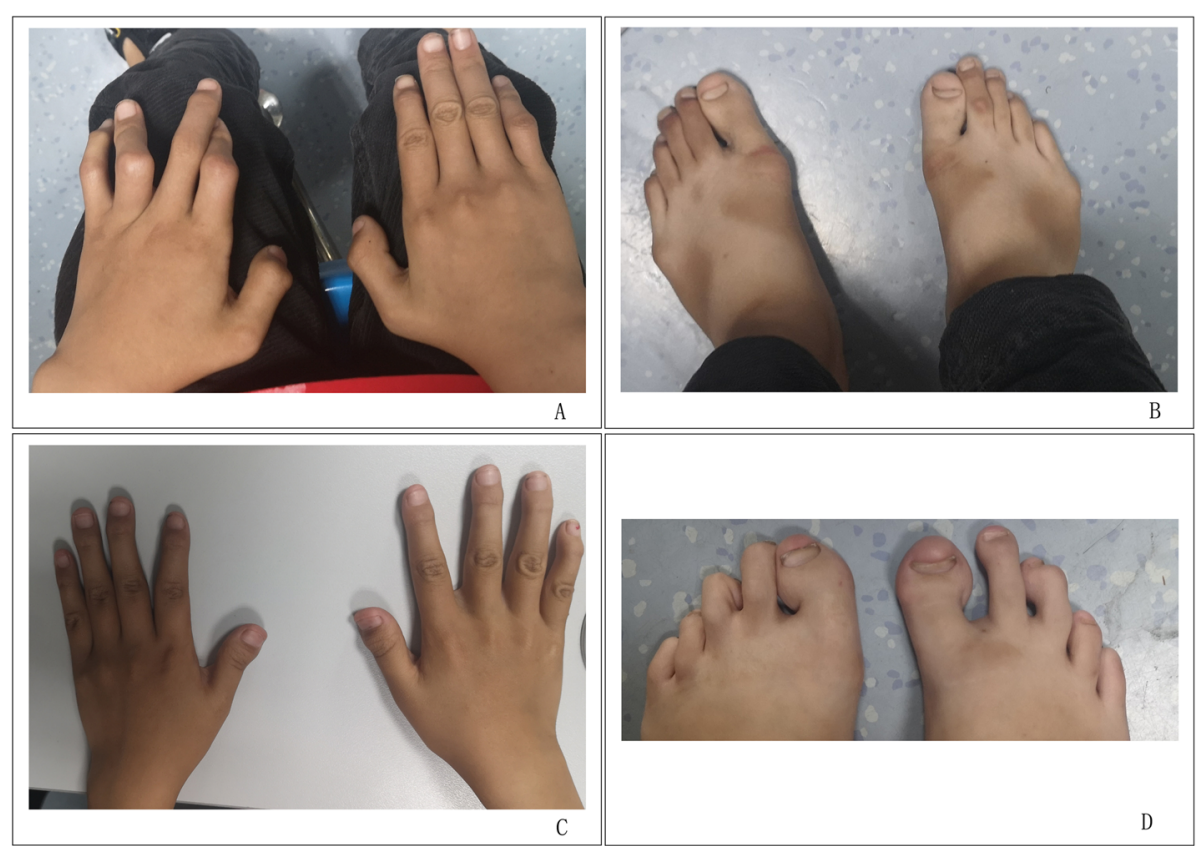

Fig. 1 The clinical photographs of the hands and feet of child 2 and 3. a and $\mathbf{b}$ : Child 2 at age 13 years, male; there were abnormal curvature of the fingers (toes) in both hands (feet); $\mathbf{c}$ and $\mathbf{d}$ : Child 3 at age 8 years, female; there were abnormal curvature of the fingers (toes) in both hands (feet)

radiographs showed bifurcation in the distal phalanx of the first finger of right hand, Metacarpophalangeal (mp) joint dislocation, distal finger joint dislocation, the bone line closure in the distal phalanx of the two hands and the advanced carpal bone ages which were the typical hand manifestations of the DBQD type 1. From clinical symptoms and radiological findings we can diagnose these two patients as DBQD type 1 .

\section{Identification of the novel compound heterozygous mutations of the CANT1 gene}

We examined the 3 children and their parents by using WES. Based on the autosomal recessive inheritance, types of the mutations and minor allele frequency, the variants were filtered. Novel compound heterozygous mutations of the CANT1 gene were found in the 3 children (c.594G > A [p.Trp198*], c.734C $>$ T [p.Pro245Leu]). Two loci were heterozygous mutations located in the second and third exons, respectively.

The mutations of the CANT1 gene were then confirmed by direct sequencing. From the sequencing results, we could see that the mutation c.594G > A of the 3 children was inherited from the mother. Mutation c.734C $>\mathrm{T}$ was inherited from the father. It could be concluded that the mutations were compound heterozygous mutations and the genetic phenotypes were co separated (Fig. 4).

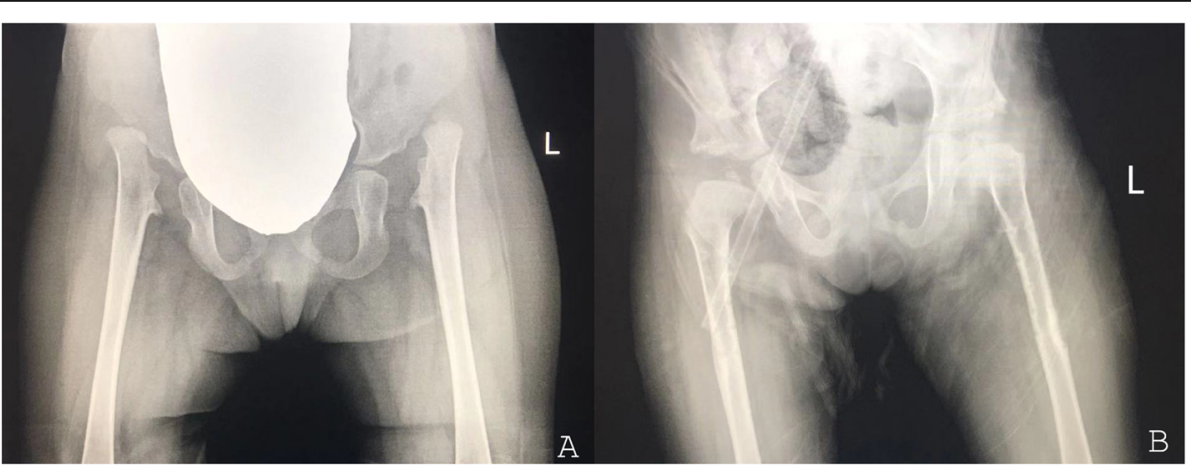

Fig. 2 Radiographs of the hip of child 3. a: Hip joint at 3 years old, Swedish key appearance could be observed; b: Hip joint after operation at 4 years old, the postoperative prognosis is good 


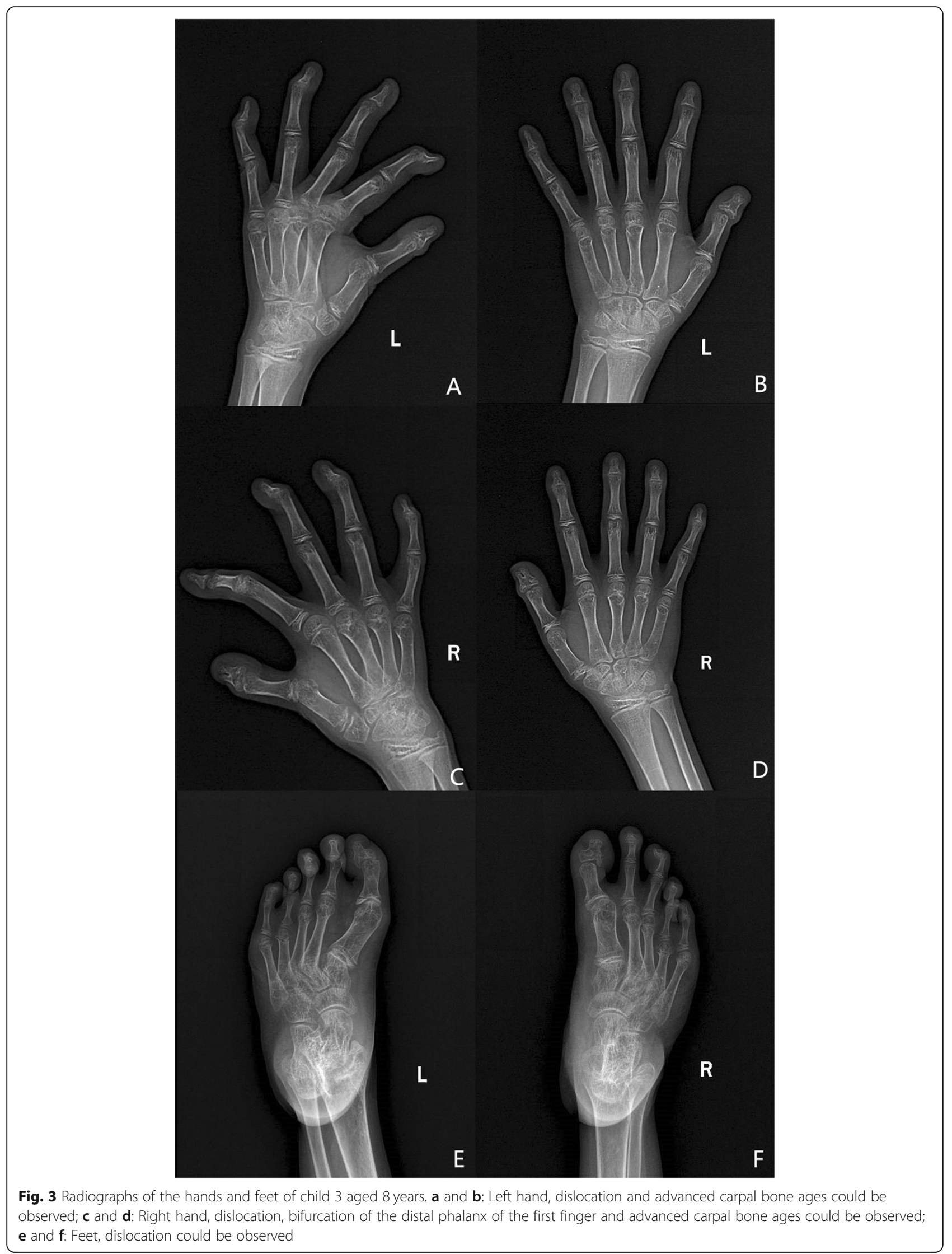




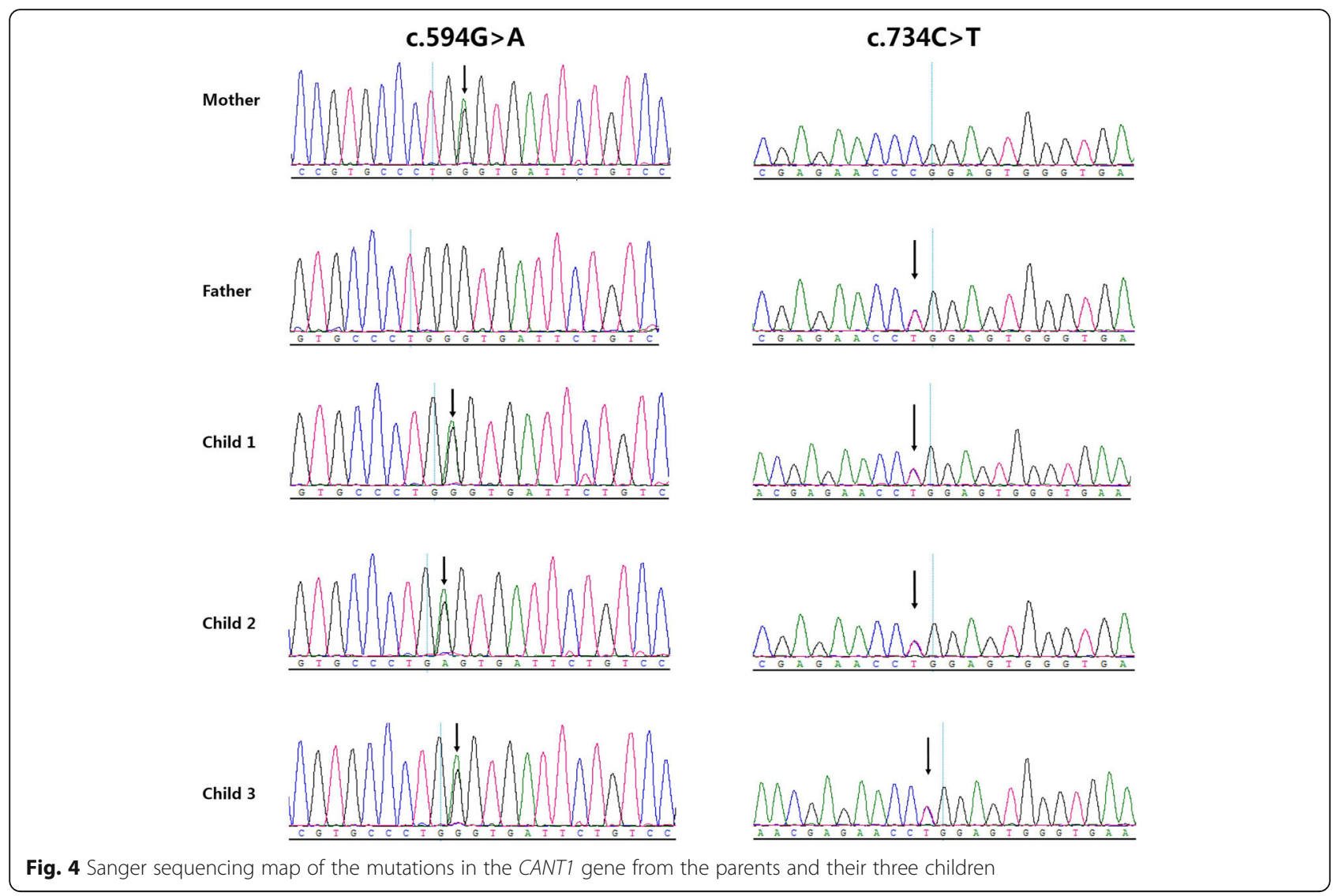

The effect of mutations on the CANT1 gene expression

The constructed eukaryotic expression vector pEGFP-C1 CANT1 wt / mut1 (c.594G > A)/mut2 (c.734C > T) was transfected into HEK293T cells for $48 \mathrm{~h}$ and then detected (Fig. 5a). Photographs were taken with a GFP fluorescence microscope, and it was found that the wild-type CANT1 protein was localized mainly in the nucleus. The GFP signal of mut1 was significantly weakened, while the GFP signal of mut 2 was significantly enhanced and obvious localization was visible in the nucleus (Fig. 5b).

qPCR was used to detect the mRNA expression of wild-type and mutant genes in HEK293T cells. The results showed that the expression of the CANT1 mut1 was significantly reduced and the expression of the CANT1 mut2 was significantly increased. It showed that the mutations affected the expression of CANT1 gene at the transcription level (Fig. 5c).

The Western blotting (WB) results were showed in Fig. 5d. Compared to the wild-type CANT1 $(72 \mathrm{kDa}$, GFP-CANT1-wt), mut1 was a nonsense mutation leading to premature termination $(49 \mathrm{kDa}, \mathrm{GFP}-C A N T 1-$ mut1), producing truncated bodies. The protein expression of mut 1 was significantly reduced; while mut 2 did not lead to premature termination $(72 \mathrm{kDa}$, GFPCANT1-mut1) and the protein expression was significantly increased.

\section{Detection of CANT1 dimerization}

The non-reducing WB method was used to detect the difference of CANT1 wt / mut2 dimerization. The constructed eukaryotic expression vector pEGFP-C1 CANT1 wt/mut2 was transfected into HEK293T cells. After $36 \mathrm{~h}$ of transfection, $2 \mathrm{mM} \mathrm{Ca}^{+}$was added to stimulate and samples were collected for WB detection. The results showed that the wild-type CANT1 had a band at 144 $\mathrm{kDa}$, indicating that dimerization could occur. The mutant CANT1 dimer was significantly reduced and even undetectable by WB (Fig. 6a). Quantitative results after scanning of gray values suggested that CANT1 mut2 could affect the occurrence of CANT1 dimers (Fig. 6b).

\section{Detection of protein secretion and nucleotide enzyme activity}

We collected the supernatants of the cells and detected the difference in the content and activity of CANT1 wt/ mut $1 /$ mut 2 protein secreted extracellularly by $\mathrm{WB}$ and molybdenum blue colorimetry. WB results showed that compared with the secreted protein of wild-type CANT1 (72 kDa, GFP-CANT1-wt), the nonsense mutation mut1 (c.594G > A) also occurred extracellular secretion, and its secretion was extremely high. The secretion of mut2 (c.734C $>$ T) was significantly reduced (Fig. 7a). The results of nucleotide enzyme activity showed that 

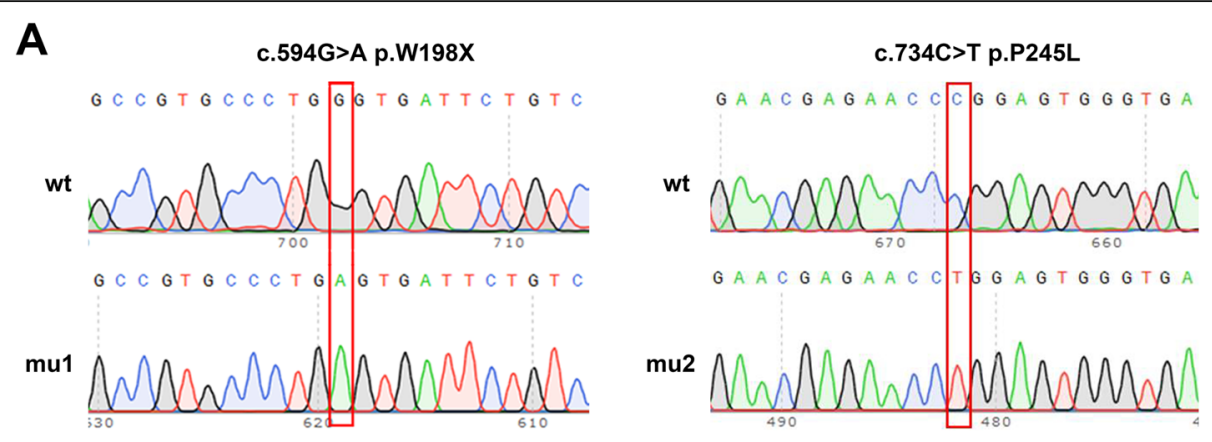

B
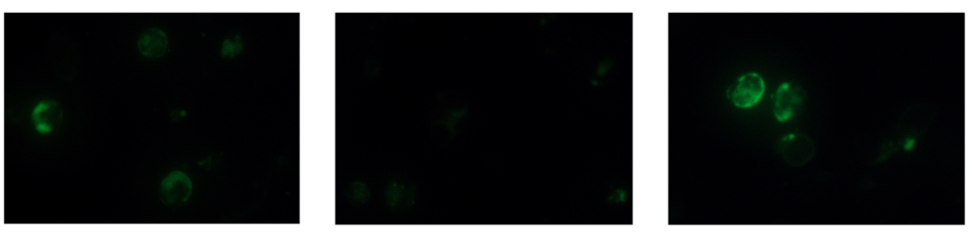

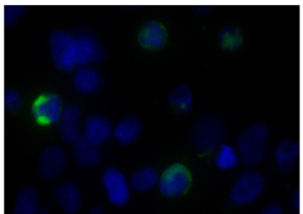

pEGFP-CANT1 ${ }^{\text {wt }}$

C

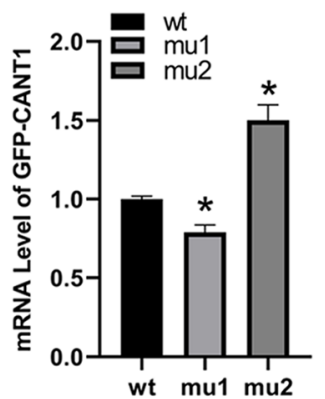

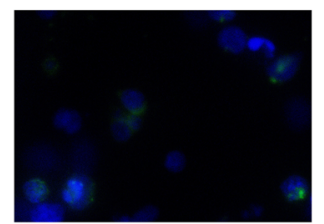

PEGFP-CANT1 ${ }^{\text {mut1 }}$

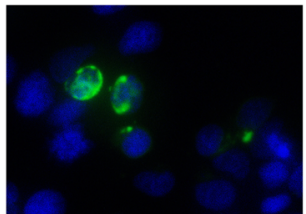

pEGFP-CANT1 $^{\text {mut2 }}$

D

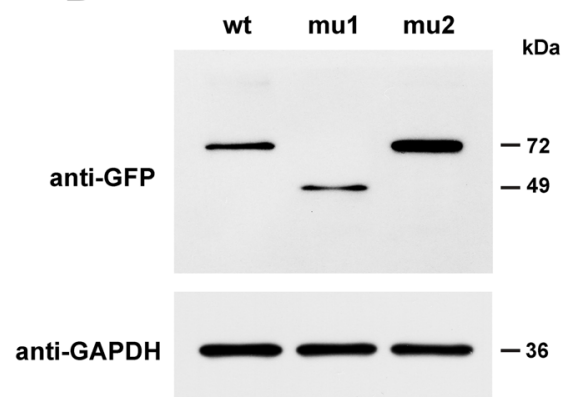

Fig. 5 Mutations c.594G > A (p.W198X) and c.734C > T (p.P245L) caused changes in the expression level of the CANT1 gene. a: Sequencing results showed that mutations c.594G > A (p.W198X) and c.734C> T (p.P245L) were successfully introduced; b: IF results (measurement scale was 40 times); c: mRNA expression detected by qPCR; $\mathbf{d}$ : CANT1 protein expression results by WB

mutations of mut1 (c.594G > A) and mut2 (c.734C > T) resulted in a significant reduction in the activity of CANT1 nucleotidease (Fig. 7b).

\section{D and 3D structure of CANT1 (Mut 2: p.Pro245Leu)}

2D structure of CANT1 (p.Pro245Leu) was showed in Fig. 8. We could see that the Pro245 residue was involved in the coil of the $2 \mathrm{D}$ structure and the prediction reliability was high. It was a highly conserved residue located on a conserved 2D fragment [13]. Mutations in this residue might affect the function of the human CANT1 protein. 3D structure of CANT1 (p.Pro245Leu) was showed in Fig. 9. On the left was the mutation sensitivity diagram of CANT1 protein (Fig. 9a). On the right was the mutation sensitivity diagram of CANT1 protein showing Pro245 position and the mutation position was shown in orange and marked with arrow (Fig. 9b). Positions were colored according to the average effect of the 20 possible mutations at that position. According to the color, we could predict whether the missense mutations in the protein were likely to have a functional/phenotypic effect. It was divided into 8 levels according to the color column in the middle of the figure, and the mutation sensitivity of Pro245 was at the 5th level. Proline was a neutral amino acid which was a cyclic sub-amino acid and its solubility in water was greater than other amino acids. Leucine was a branched-chain amino acid which was almost insoluble in water. Changes in amino acid structure and hydrophilicity might affect the protein activity. 


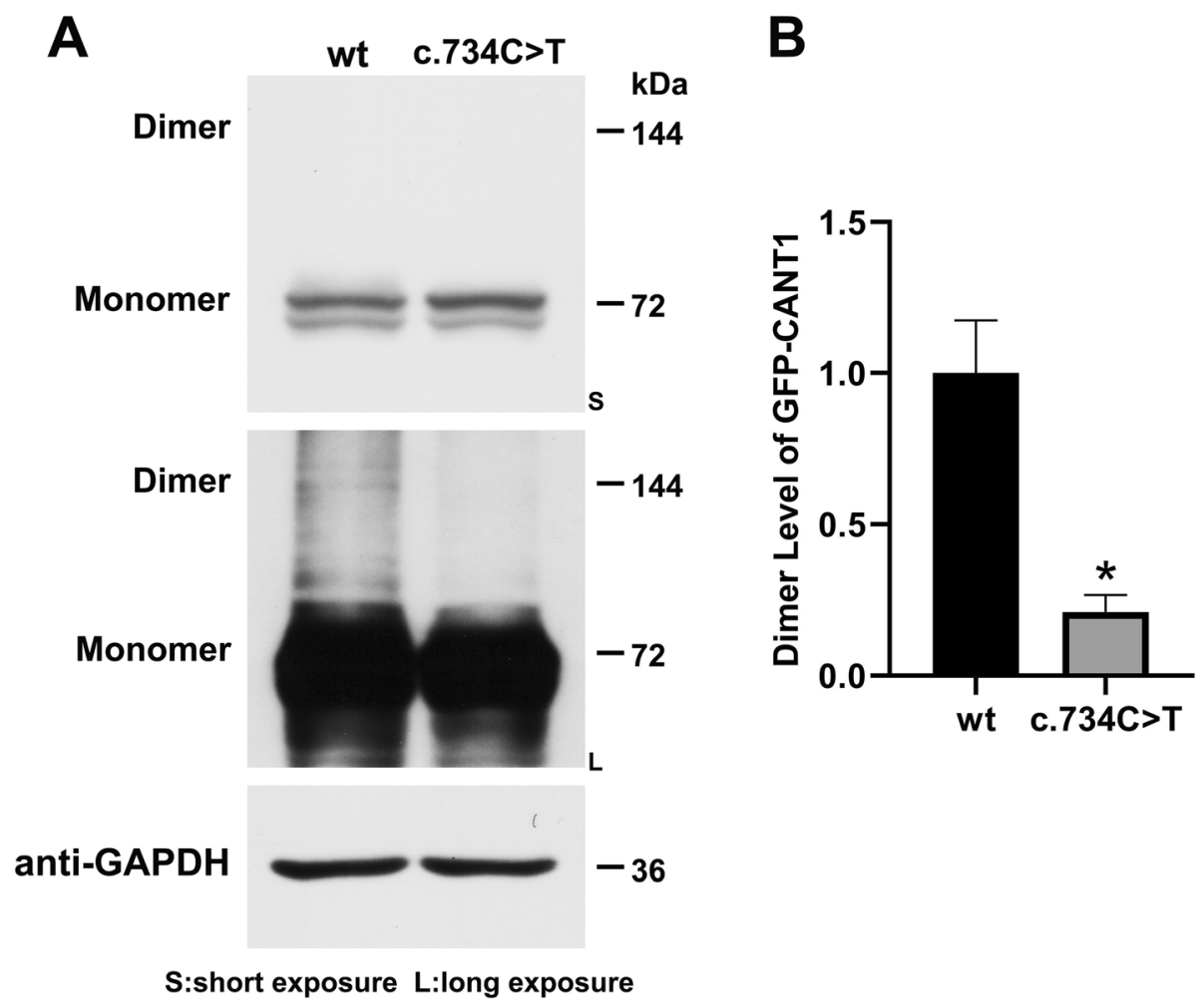

Fig. 6 The mutation c.734C > T (p.P245L) caused the CANT1 dimer to depolymerize. a: the expression of CANT1 dimerized protein was detected by WB. $\mathbf{b}$ : the WB gray scan results of CANT1 dimerized protein expression

\section{Discussion}

DBQD was classified as the multiple dislocation group according to nosology and classification of genetic skeletal disorders [1]. Clinical symptoms included multiple joint dislocations, joint laxity, scoliosis/lordosis, severe pre and postnatal growth retardation. As has been noted, DBQD type 1 and type 2 were distinguished according to the presence or absence of unique hand abnormalities [6]. The Kim variants were characterized by accelerated carpal bone ages, short metacarpals and elongated phalanges (nearly equal length of the second to fifth fingers). Severe precocious ostarthritis of the hands and spine was a major characteristic finding of the Kim variants [7]. As far as I known, 36 cases of DBQD have been reported and the CANT1 mutations were found in almost all the DBQD type 1 patients [4, 9, 10, 14-18]. There were 27 distinct CANT1 mutations were found, including 12 missense mutations, 11 exon region frame shift
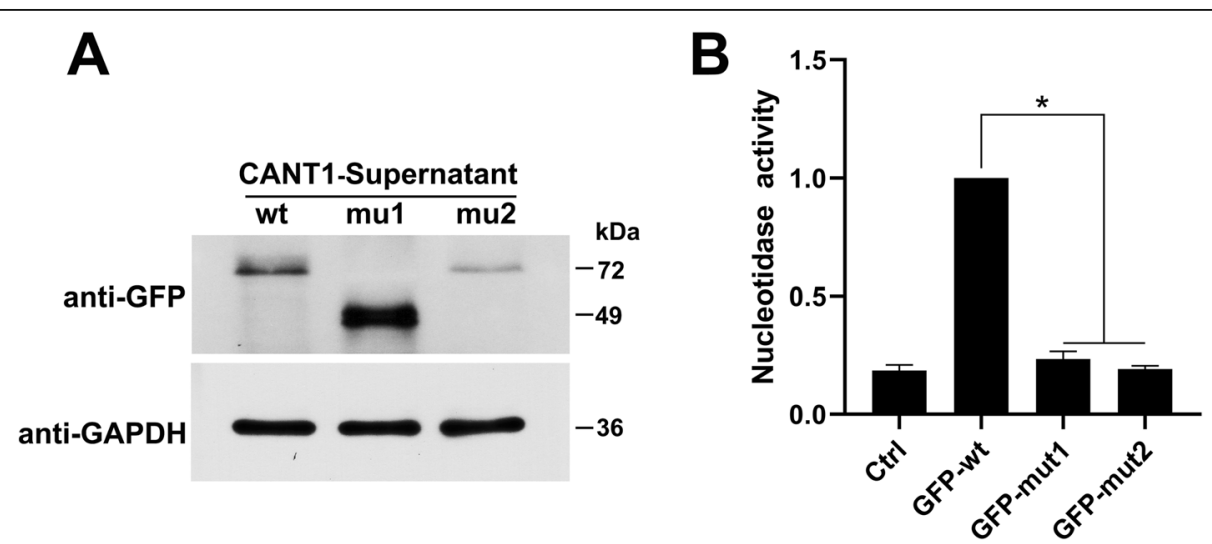

Fig. 7 The two mutations affect the extracellular secretion and nucleotide activity of CANT1. A: the expression of the extracellular secreted CANT1 protein was detected by WB; B: the result of CANT1 nucleotide enzyme activity 


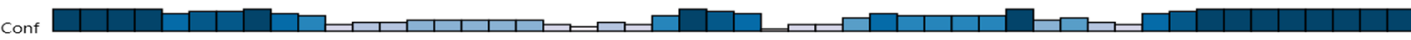

Cart

Pred C C C C C C C C C C C C C C C C C C CEE C C C C C C C C C C C C C C C H H H H H H H H AA MP VQLSE HPE WNES MHSLRI S VGGLPVLAS MT KAADPRFRPR WKVILTFF

20

30

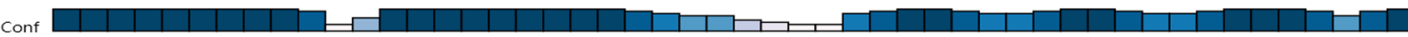

Cart

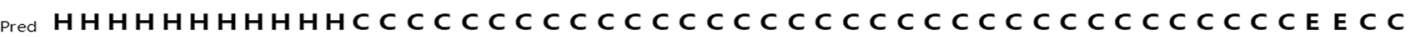

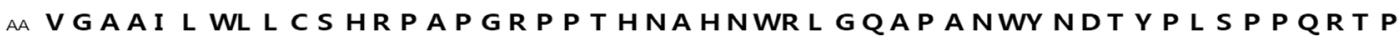

cont

Cart

Pred C C CE E E E E E E E ECCCCCCCCCCCE E E E E E E E E E E E E C C CEE E E E C C AA AGI RYRI AVI ADLDTESRAQEENT WF Y K K K YLTLS DSGDKVAVEWDKD

130

Conf

Cart

Pred CE E E E E C C C C C C C C C C C E E E C E E E E C C C CEE E E E C C CE E E E AA HGVL E HLAE K GRGMELS DL I V NGKL Y S VDDRTGVVYQIEGS KAVP WVI

160

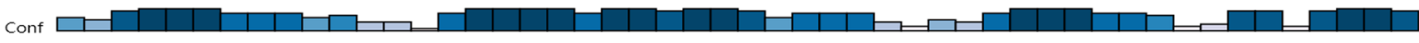

Cart

Pred C C C C C C C C C C C E E E E E E C C E E E E C C C CE E C C C E E ECCEE E E AA L S DGDGT VE KG F K AE WLAVKDERL Y VGGL G KE WT T T T DVVNENPEWVKV

$$
230 \quad 240 \quad \text { | } 250
$$

Conf

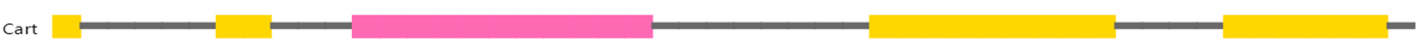

Pred EC C C C C E E C C H H H H H H H H C C C C C CEE E E E E E C C C E E E E E C AA VGYKGS VDHENWVSNYNALRAAAGI QPPGYLI HESACWSDTL QR WFFLPR

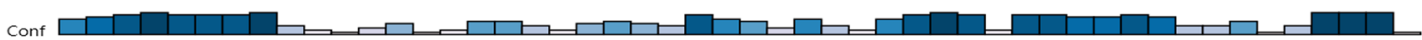

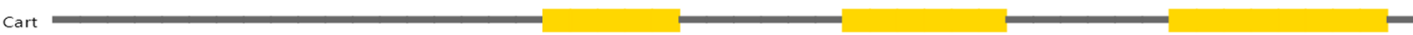

Pred CCCCCCCCCCCCCCCCCCE E EEECCCCCCEEEE EECCCCCCEEE EEEEEC AA RASQERYSEKDDERKGANLLLSASPDFGDIAVSHVGAVVPTHGFS SF KFI

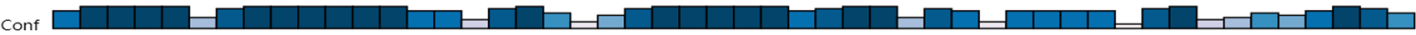

Cart

Pred C C C C C E E E E E E E E ECCCCE E E E E E E C C CE C C E E E C CEE CE E E AA PNTDDQI I VALKSEEDSGRVASYI MAFTLDGRFLLPETKI GSVKYEGIEF

Conf $\square$

Cart $=$

Pred C

AA I

$$
\begin{aligned}
& \begin{array}{llllll}
\text { Legend: } & 410 & 420 & 430 & 440 & 450
\end{array} \\
& \text { Strand Conf: }-1+n+\text { Confidence of prediction } \\
& \text { Helix Cart: 3-state assignment cartoon } \\
& \begin{array}{ll}
\text { - Coil } & \text { Pred: 3-state prediction } \\
& \text { AA: Target Sequence }
\end{array}
\end{aligned}
$$

Fig. 8 2D structure of CANT1 (p.Pro245Leu), and arrow indicated mutation position 

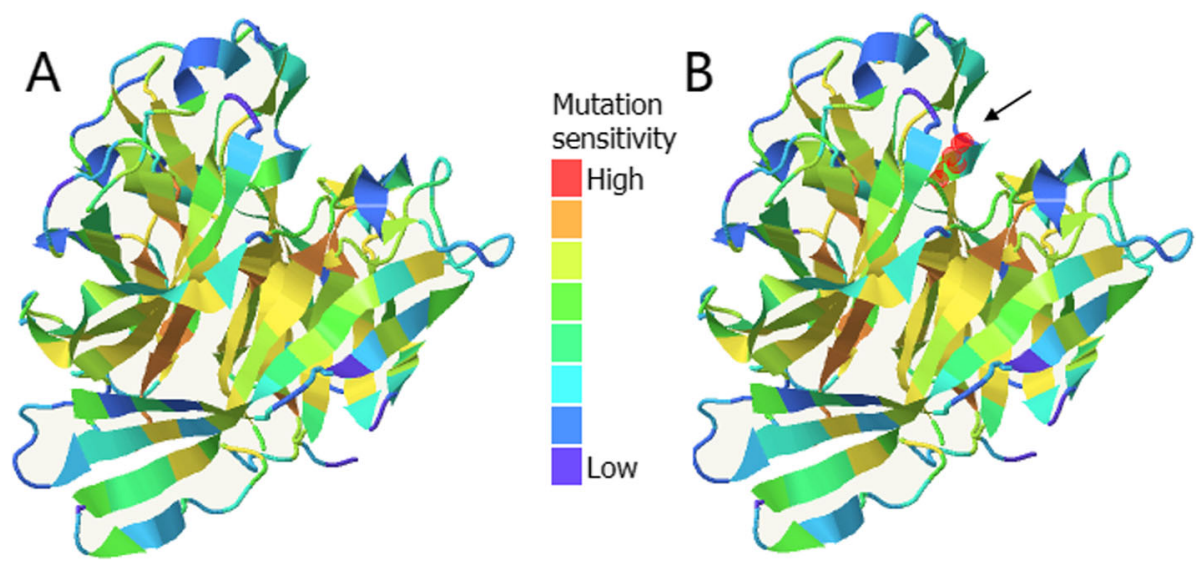

Fig. 9 3D structure of CANT1 (p.Pro245Leu). a: the mutation sensitivity diagram of CANT1 protein; b: the mutation sensitivity diagram of CANT1 protein showing Pro245 position in orange and marked with arrow

mutations, 2 nonsense mutations, 1 intron frame shift mutation and 1 splice site mutation. All three types of DBQD could be observed only in the Turkish population. Until now, the Kim variant has only appeared in Japan, Korea and India which belonged to Asia and Turkish population. CANT1 mutations were supported as the causes of DBQD type 1 patients, Kim variant and atypical DBQD type 2 (only two cases). There were still some unknown molecular bases of DBQD type 2. It was important to correctly diagnose and type the DBQD based on the patient's clinical symptoms rather than relying solely on genetic mutation information.

Here we reported two novel CANT1 mutations (compound heterozygous) in three children from a family of Chinese origin. Two of them (child 2 and 3) could be diagnosed as DBQD type 1 and the remaining child (child 1) could not currently be diagnosed with clinical symptoms. The parents have proven to be the biological parents of the children by short tandem repeats (STR). The STR results were showed in Supplementary Fig. 1. We found cardiac mitral valve prolapse in both patients, which has not been found in previous reports of DBQD type 1 patients. Unfortunately, we did not get more clinical symptoms and physical examination results for the child 1. According to the parents' statement, no symptoms such as joint dislocation and cardiac abnormalities occurred, and there was no history of surgery of child 1. According to previous related reports, we believed that the mutations of CANT1 might to be the cause of the disease in these two patients. In this case, it was particularly important to study the function of the two mutations of CANT1.

Until now, the molecular mechanism of DBQD caused by CANT1 gene was less studied. The CANT1 gene could form 12 transcript variants. The gene of transcript variant 1 was formed by exon $1,2,3$ and 4 which had 8 nucleotidase conserved regions (NCR) [13]. In this study, novel compound heterozygous mutations of the CANT1 gene were found in the family (mut1: c.594G > A [p.Trp198*], mut2: c.734C $>\mathrm{T}$ [p.Pro245Leu]) and located in the second and third exons, respectively. Mut1 was located in NCR3 (Exon 2) which was highly conserved and was the same in all the selected 11 sequences (Supplementary Fig. 2 A and B). Mut1 caused the formation of a stop codon, and the protein was truncated at position 198 which will likely affect NCR3-8, destroy important conserved structures of the protein, and might have an impact on the protein function. Mut2 was located in NCR5 (Exon 3) which had the same residue in all the selected 11 sequences and was as conservative as mut1 (Supplementary Fig. 2 A and B). In addition, a homozygous mutation of c.734 delC [p.P245RfsX3] has been found in a DBQD type 1 patient from Turkey and the mutation was lethal [9]. Mut2 was also occurred at this position which might mean that this locus was important for the function of the protein.

CANT1 was a soluble nucleotide enzyme belonged to apyrase family which could preferentially hydrolyze UDP next GDP and UTP $[19,20]$. However, the exact function of the protein was still unknown [21]. In order to further understand the function of human CANT1 protein, we constructed eukaryotic expression vector pEGFP-C1 CANT1 wt/mut1 (c.594G > A)/mut2 (c.734C > T) and transfected into HEK293T cells. We studied the effect of mutations on the CANT1 gene expression, protein secretion and nucleotide enzyme activity. For mut1, it could be seen from the results that truncated protein was formed, the amount of distribution around the nucleus and activity were decreased and extracellular secretion was increased. Previous research showed that the activity of this family was strictly dependent on calcium, and the calcium could cause conformational changes of the secreted, soluble 
human nucleotidase protein [22]. The truncated protein formed by mut1 might lose the critical calcium-binding region for activity which was also critical region responsible for forming dimerization through covalent binding, but retain the region responsible for extracellular secreted. For mut2, there were some phenomena different from mut1. It could be seen that the expression was increased, dimerization was disappeared, the amount of distribution around the nucleus was increased, and extracellular secretion and activity were decreased. The increase in expression might be due to the disappearance of dimerization, rather than a true increase in expression. Pro245 might be a critical region for calcium binding. And the mutation affected the conformation of the protein, leading to changes in its secretion and activity. In addition, the prediction of the 2D and 3D structure of CANT1 showed that Pro245 was in an important 2D structure (coil) and was located in a superficial position of the protein. The highly hydrophilic cyclic amino acid proline was replaced by the low hydrophilic branched chain amino acid leucine which will definitely affect the function of the protein.

Previous studies have shown that mutations in the CANT1 gene may be related to impaired endoplasmic reticulum function [9], and may also affect Golgi function and affect proteoglycan synthesis [4]. Other study has shown that CANT1 was found to be a target gene of the DREAM protein involved in the process of protein folding and degradation [23]. In short, CANT1 gene mutations might cause a defect in the CANT1 protein by affecting CANT1 protein secretion, conformation, etc. and affect the protein biosynthesis process. Recently, CANT1 knock-in and knock-out mice have been successfully prepared and the DBQD type 1 phenotype was recapitulated [24]. This provided individual phenotypic evidence of the important role of the gene in cartilage ossification and the synthesis of cartilage proteoglycan.

\section{Conclusions}

In this study, we reported two novel CANT1 mutations in three children from one family. It was worth pointing out that two of the three could be diagnosed as typical DBQD type 1 according to their clinical symptoms. At the same time, a new symptom (mitral valve prolapse) different from previous reports was observed. However, the other child only had height restrictions with a height of $142 \mathrm{~cm}$ in her 16 years who couldn't be diagnosed as DBQD based on our clinical data. Based on the above, we could consider that the mutations of the CANT1 gene were the cause of the two cases of DBQD type 1 in this study. We haven't been able to give a reasonable explanation as to why the child 1 did not have symptoms of DBQD type 1. The substrates (UDP, GDP and UTP) of the CANT1 protein were involved in the functional regulation of several important signaling molecules, which showed not only the importance of CANT1 but also the complexity of its role. This might explain the genetic heterogeneity present in this study. The genetic and phenotype of three children with the same genetic background need to be further studied, and the pathogenesis of CANT1 may be more complicated.

\section{Materials and methods}

\section{Patients and clinical assessment}

The patients were identified and collected through clinical genetics counseling services at the clinic of Henan Provincial People's Hospital. The parents on behalf of the children signed the informed consent for the study. The study was approved by the human and ethics committee of Henan Provincial People's Hospital.

There are 5 members in this family, including parents with normal phenotype and 3 children with abnormal phenotype. The healthy parents of Chinese Han descent were not close relatives and had no history of adverse contact during pregnancy. The clinical manifestations and radiological findings of the 3 children were summarized in Table 1. According to the diagnostic criteria for DBQD type 1 and the clinical manifestations of the children, children 2 and 3 could be diagnosed as DBQD type 1. The clinical manifestations for these two patients consistent with DBQD type 1 included: pre and postnatal growth retardation, dislocation of joint, Swedish key appearance of the proximal femur and characteristic hand anomalies (Figs. 1, 2 and 3).

\section{DNA extraction, whole exome sequencing and data analysis}

The peripheral blood samples were obtained from the couple and their three children. Genomic DNA was extracted from $500 \mu \mathrm{l}$ peripheral bloods using a standard commercial kit (TIANGEN, Beijing, China) following the manufacturer's instructions. The quantity of the DNA samples was determined using Qubit dsDNA HS assay kit on Qubit 3.0 (Invitrogen Co., California, USA).

Whole Exome Sequencing (WES) was conducted using the AIExomeV2 kit (iGeneTech Co., Beijing, China). According to the manufacturer's standard operation protocol, the whole exon region was enriched by liquid phase probe method and sequenced on Illumina Nova sequence platform (Illumina, Inc., California, USA). 1\% was used as Minor allele frequency (MAF) threshold in this study. The depth used in the WES was 100x and the coverage of targeted exons was $99 \%$.

\section{Mutation confirmation}

In order to determine the mutations and identify whether the mutations co-segregated with the disease phenotype the 5 family members were sequenced and analyzed using ABI3500 Genetic Analyzer and 
Sequencing Analysis software (Applied Biosystems, Foster City, CA, USA). Specific sequences containing the two mutations were amplified and Primer 5 software was used for designing the primers. The primers were showed in Supplementary Table 1.

\section{CDNA cloning and construction of expression vectors}

Target cDNA synthesis was completed by Wuhan Shishi Biopharm Co. Ltd. using gene splicing by overlap extension PCR method (SOE PCR). Using human CANT1 cDNA as a template, an expression vector was constructed: the entire length of the CANT1 cDNA was cloned into the pEGFP-C1 plasmid through restriction sites Xho I and BamH I to construct a recombinant vector pEGFP-C1 CANT1 wt (wild type) (Wuhan Shishi Biopharm Co., Wuhan, China). Using site-directed mutagenesis, the mutations c.594G > A [p.Trp198*] and c.734C $>\mathrm{T}$ [p.Pro245Leu] were introduced into the above-mentioned recombination vectors, respectively, and the recombinant vectors pEGFP-C1 CANT1 mut 1 and pEGFP-C1 CANT1 mut 2 were constructed. This set of vectors expressed the fusion protein GFP CANT1.

\section{Cell transfection and immunofluorescence (IF) staining}

HEK293T cells were cultured in DMEM medium (Gibco, Thermo Fisher) containing 10\% fetal bovine serum, and the constructed wild-type and mutant eukaryotic recombinant expression vectors were transiently transfected into HEK293T cells according to the liposome instructions of Lipo2000 (YEASEN, Shanghai, China). Forty-eight hours after transfection, wild-type and mutant fluorescent pictures were taken and qPCR tests were performed after collection. SYBR Green Realtime PCR Master Mix was used for qPCR (TOYOBO Co., Shanghai, China).

\section{RNA analysis}

Cell samples were collected $48 \mathrm{~h}$ after transfection of wildtype and mutant recombinant expression vectors. Total RNA was extracted by Trizol method (Invitrogen, Gaithersburg, MD, USA). cDNA synthesis was performed using a commercialization Kit according to the instructions (Takara, Dalian, China). And the expression difference of wild-type and mutant target genes was detected by qPCR by ABI StepOne Real-Time PCR system (ABI, Vernon, USA). SYBR Green Realtime PCR Master Mix was used for qPCR (TOYOBO Co., Shanghai, China).

\section{Western blotting (WB)}

After $36 \mathrm{~h}$ of the wild-type and mutant recombinant expression vectors transfection, the medium was replaced with serum-free medium Opti-MEM and the culture was continued for $36 \mathrm{~h}$ to collect the supernatant and cell pellet. Use Amicon ${ }^{\circ}$ Ultra-4 Centrifugal Filters to process the cell supernatant at $4{ }^{\circ} \mathrm{C}$ and $3000 \mathrm{rpm}$, and concentrate $2 \mathrm{~mL}$ of the supernatant to $200 \mathrm{ul}$ to extract the secreted proteins. RIPA lysate was used to extract total protein from the cell pellet. The BSA kit was used to determine the protein concentration, followed by protein denaturation treatment, and the WB method was used to detect the difference in protein expression of wild-type and mutants and the degree of dimerization of the target proteins.

Non-reduced WB was used in this study. The method of non-reduced WB was basically the same as that of conventional WB, but fresh and sufficient protease inhibitor (Pierce) was added to the cell lysate, and the reducing agent components such as SDS must be removed from the cell lysate, loading buffer, and PAGE gel.

\section{Nucleotidase activity assay}

The treatment of cell culture supernatant was as follows. Concentrate $1 \mathrm{~mL}$ of the supernatant to $100 \mathrm{ul}$ to extract the secreted proteins. The 100ul supernatant was diluted 10 times with $40 \mathrm{mM}$ succinate buffer $(\mathrm{pH} 6.5$, containing $4 \mathrm{mM} \mathrm{CaCl}{ }_{2}, 2 \mathrm{mM} \mathrm{UDP}$ ), and incubated at $37^{\circ} \mathrm{C}$ for $1 \mathrm{~min}$. The inorganic phosphorus detection kit was used to detect the free phosphorus Pi content using ferrous sulfate molybdenum blue microplate method according to the instructions, and the CANT1 nucleotide enzyme activity was calculated.

\section{Prediction of 2D and 3D structure of CANT1 (Mut 2:p.Pro245Leu)}

The homologous sequence of human CANT1 protein was extracted from NCBI-Homologene Database and used an online website PredictProtein (http: //www.predactprotein) predicted the 2D structure of CANT1 [25]. The online website Phyre (www.sbg.bio.ic.ac.uk/phyre) was used for predicting the 3D structure of CANT1 [26].

\section{Supplementary information}

Supplementary information accompanies this paper at https://doi.org/10. 1186/s13023-020-01492-8.

Additional file 1: Supplementary Figure 1. The STR results of the 5 family members. A: Mother; B: Father; C: Child 1; D: Child 2; E: Child 3.

Additional file 2: Supplementary Figure 2. The gene structure and the genomic alignment of the CANT1 gene. A: The diagram of ExonIntron-Mutation of the CANT1 gene; B: The genomic alignment for mut 1 and mut 2 conservation of 11 CANT1 Homologous sequences.

Additional file 3: Supplementary Table 1. The sequence of the primers used in mutation confirmation.

Abbreviations

DBQD: Desbuquois dysplasia; CANT1: Calcium activated nucleotidase 1; IF: Immunofluorescence; WB: Western blotting

\section{Acknowledgements}

We would like to thank the patients and their parents for participation in this study. 


\section{Authors' contributions}

HDW and BFZ contributed to the research design, conducted the study and wrote the manuscript. LJG was responsible for sample collection, counseling, clinical examination and data analysis. ZQF and DWZ were responsible for sample collection, clinical examination and conducted the experiments. MTZ, YG and CLC conducted the experiments and data analysis. All authors have read and approved the final manuscript.

\section{Funding}

This study was supported by the National Natural Science Foundation of China (81501336), the Science and Technology Research Project of Henan Province (172102310295), Opening Foundation of National Health Commission Key Laboratory of Birth Defects Prevention (ZD202006) and Henan Medical Science and technology project (2018020389).

\section{Availability of data and materials}

All data could acquire from the corresponding author if reasonably required.

\section{Ethics approval and consent to participate}

The study was approved by the human and ethics committee of Henan Provincial People's Hospital.

\section{Consent for publication}

All participants gave their informed consent for the study.

\section{Competing interests}

The authors declare that they have no competing interests.

\section{Author details}

'Medical Genetic Institute of Henan Province, Henan Provincial People's Hospital, Zhengzhou University People's Hospital, Zhengzhou 450003, People's Republic of China. ${ }^{2}$ National Health Commission Key Laboratory of Birth Defects Prevention, Henan Key Laboratory of Population Defects Prevention, Henan Institute of Reproduction Health Science and Technology, Zhengzhou 450014, People's Republic of China. ${ }^{3}$ Clinical Research Center of Shaanxi Province for Dental and Maxillofacial Diseases, College of Stomatology, Xi'an Jiaotong University, Xi'an 710004, People's Republic of China. ${ }^{4}$ College of Forensic Science, Xi'an Jiaotong University Health Science Center, Xi'an Jiaotong University, Xi'an, Shaanxi 710061, People's Republic of China. ${ }^{5}$ Department of Urology, The First People's Hospital of Zhengzhou, Zhengzhou 450004, People's Republic of China. ${ }^{6}$ Zhengzhou Orthopaedic Hospital, Zhengzhou 450052, People's Republic of China.

\section{Received: 13 February 2020 Accepted: 5 August 2020}

\section{Published online: 09 September 2020}

\section{References}

1. Mortier GR, Cohn DH, Cormier-Daire V, Hall C, Krakow D, Mundlos S, Nishimura G, Robertson S, Sangiorgi L, Savarirayan R, Sillence D, Superti-Furga A, Unger S, Warman ML. Nosology and classification of genetic skeletal disorders: 2019 revision. Am J Med Genet A. 2019 Dec;179(12):2393-419.

2. Hall BD. Lethality in Desbuquois dysplasia: three new cases. Pediatr Radiol. 2001;31(1):43-7.

3. Desbuquois G, Grenier B, Michel J, Rossignol C. Nanisme chondrodystrophique avec ossification anarchique et polymalformations chez deux soeurs. Arch Fr Pediatr. 1966;23:573-87.

4. Nizon M, Huber C, De Leonardis F, Merrina R, Forlino A, Fradin M, Tuysuz B, Abu-Libdeh BY, Alanay Y, Albrecht B, Al-Gazali L, Basaran SY, Clayton-Smith J, Désir J, Gill H, Greally MT, Koparir E, van Maarle MC, MacKay S, Mortier G, Morton J, Sillence D, Vilain C, Young I, Zerres K, Le Merrer M, Munnich A, Le Goff C, Rossi A, Cormier-Daire V. Further delineation of CANT1 phenotypic spectrum and demonstration of its role in proteoglycan synthesis. Hum Mutat. 2012;33(8):1261-6.

5. Baynam G, Kiraly-Borri C, Goldblatt J, Dickinson JE, Jevon GP, Overkov A. A recurrence of a hydrop lethal skeletal dysplasia showing similarity to Desbuquois dysplasia and a proposed new sign: the upsilon sign. Am J Med Genet A. 2010;152A(4):966-9.

6. Faivre L, Cormier-Daire V, Eliott AM, Field F, Munnich A, Maroteaux P, Le Merrer M, Lachman R. Desbuquois dysplasia, a reevaluation with abnormal and "normal" hands: radiographic manifestations. Am J Med Genet A. 2004;124A(1):48-53.
7. Kim OH, Nishimura G, Song HR, Matsui Y, Sakazume S, Yamada M, Narumi Y, Alanay Y, Unger S, Cho TJ, Park SS, Ikegawa S, Meinecke P, Superti-Furga A. A variant of Desbuquois dysplasia characterized by advanced carpal bone age, short metacarpals, and elongated phalanges: report of seven cases. Am J Med Genet A. 2010;152A(4):875-85.

8. Faivre L, Le Merrer M, Al-Gazali LI, Ausems MG, Bitoun P, Bacq D, Maroteaux P, Munnich A, Cormier-Daire V. Homozygosity mapping of a Desbuquois dysplasia locus to chromosome 17q25.3. J Med Genet. 2003;40(4):282-4.

9. Huber C, Oulès B, Bertoli M, Chami M, Fradin M, Alanay Y, Al-Gazali LI, Ausems MG, Bitoun P, Cavalcanti DP, Krebs A, Le Merrer M, Mortier G, Shafeghati Y, Superti-Furga A, Robertson SP, Le Goff C, Muda AO, PaterliniBréchot $P$, Munnich A, Cormier-Daire V. Identification of CANT1 mutations in Desbuquois dysplasia. Am J Hum Genet. 2009;85(5):706-10.

10. Singh A, Kim OH, lida A, Park WY, Ikegawa S, Kapoor S. A novel CANT1 mutation in three Indian patients with Desbuquois dysplasia Kim type. Eur 」 Med Genet. 2015 Feb;58(2):105-10,

11. Bui C, Huber C, Tuysuz B, Alanay Y, Bole-Feysot C, Leroy JG, Mortier G, Nitschke P, Munnich A, Cormier-Daire V. XYLT1 mutations in Desbuquois dysplasia type 2. Am J Hum Genet. 2014;94(3):405-14.

12. Guo L, Elcioglu NH, lida A, Demirkol YK, Aras S, Matsumoto N, Nishimura G, Miyake N, Ikegawa S. Novel and recurrent XYLT1 mutations in two Turkish families with Desbuquois dysplasia, type 2. J Hum Genet. 2017;62(3):447-51.

13. Yang M, Kirley TL. Site-directed mutagenesis of human soluble calciumactivated nucleotidase 1 (hSCAN-1): identification of residues essential for enzyme activity and the $\mathrm{Ca}(2+)$-induced conformational change. Biochemistry. 2004;43(28):9185-94.

14. Faden M, Al-Zahrani F, Arafah D, Alkuraya FS. Mutation of CANT1 causes Desbuquois dysplasia. Am J Med Genet A. 2010;152A(5):1157-60.

15. Laccone F, Schoner K, Krabichler B, Kluge B, Schwerdtfeger R, Schulze B, Zschocke J, Rehder H. Desbuquois dysplasia type I and fetal hydrops due to novel mutations in the CANT1 gene. Eur J Hum Genet. 2011;19(11):1133-7.

16. Inoue S, Ishii A, Shirotani G, Tsutsumi M, Ohta E, Nakamura M, Mori T, Inoue T, Nishimura G, Ogawa A, Hirose S. Case of Desbuquois dysplasia type 1: potentially lethal skeletal dysplasia. Pediatr Int. 2014;56(4):e26-9.

17. Ma J, Yang Y, Zhang K, Huang Y, Lyu Y, Gao M, Gai Z, Liu Y. Analysis of CANT1 gene variant in a girl with Desbuquois dysplasia type I. Zhonghua $Y_{i}$ Xue Yi Chuan Xue Za Zhi. 2019:36(12):1206-9.

18. Lam WF, Chan HB, Sillence DO. Desbuquois syndrome: clinical and radiological report of the first two Chinese cases from a consanguineous family. J Paediatr Child Health. 2003;39(9):707-12.

19. Murphy DM, Ivanenkov W, Kirley TL. Bacterial expression and characterization of a novel, soluble, calcium-binding, and calcium-activated human nucleotidase. Biochemistry. 2003;42(8):2412-21.

20. Smith TM, Hicks-Berger CA, Kim S, Kirley TL. Cloning, expression, and characterization of a soluble calcium-activated nucleotidase, a human enzyme belonging to a new family of extracellular nucleotidases. Arch Biochem Biophys. 2002;406(1):105-15.

21. Lecca D, Ceruti S. Uracil nucleotides: from metabolic intermediates to neuroprotection and neuroinflammation. Biochem Pharmacol. 2008;75(10):1869-81.

22. Yang M, Horii K, Herr AB, Kirley TL. Calcium-dependent dimerization of human soluble calcium activated nucleotidase: characterization of the dimer interface. J Biol Chem. 2006;281(38):28307-17.

23. Cali T, Fedrizzi L, Ottolini D, Gomez-Villafuertes R, Mellström B, Naranjo JR, Carafoli E, Brini M. Ca2+-activated nucleotidase 1, a novel target gene for the transcriptional repressor DREAM (downstream regulatory element antagonist modulator), is involved in protein folding and degradation. J Biol Chem. 2012;287(22):18478-91.

24. Paganini C, Monti L, Costantini R, Besio R, Lecci S, Biggiogera M, Tian K, Schwartz JM, Huber C, Cormier-Daire V, Gibson BG, Pirog KA, Forlino A, Rossi A. Calcium activated nucleotidase 1 (CANT1) is critical for glycosaminoglycan biosynthesis in cartilage and endochondral ossification. Matrix Biol. 2019;81:70-90.

25. Buchan DWA, Jones DT. The PSIPRED protein analysis workbench: 20 years on. Nucleic Acids Res. 2019;47(W1):W402-7.

26. Kelley LA, Mezulis S, Yates CM, Wass MN, Sternberg MJ. The Phyre2 web portal for protein modeling, prediction and analysis. Nat Protoc. 2015;10(6):845-58.

\section{Publisher's Note}

Springer Nature remains neutral with regard to jurisdictional claims in published maps and institutional affiliations. 